\title{
Higher mortality among ACS patients in Finland than in Norway: Do differences in acute services and scale effects in hospital treatment explain the variation?
}

\author{
TRON ANDERS MOGER ${ }^{1}$ \\ UNTO HÄKKINEN ${ }^{2}$ \\ TERJE P. HAGEN ${ }^{1, *}$ \\ ${ }^{1}$ Department of Health Management and Health Economics, University of Oslo, Norway \\ ${ }^{2}$ National Institute of Health and Welfare, Helsinki, Finland
}

\begin{abstract}
Mortality following hospital treatment in Finland and Norway is similar for major diseases, with acute coronary syndrome (ACS) as an important exception. For ACS, the mortality is significantly higher in Finland than in Norway. We study whether a decentralized structure with reduced emergency preparedness and smallscale production in Finland vs. a centralized structure with large percutaneous coronary intervention (PCI) departments performing acute services 24/7 in Norway explains the country differences in mortality. For patients discharged with acute myocardial infarction (International Classification of Diseases - ICD-10 I21 and I22) and unstable angina pectoris (ICD-10 I 20.0), data from the hospital discharge registers for 1 Jan. 2009-30 Nov. 2014 was linked with socio-demographic and regional variables, variables describing distances to hospitals, and with data from causes of death registers in Norway and Finland. Variables relating to hospital system and organization of care were included as independent variables in logistic regression analyses. Marginal mortality differences between the countries for different categories of the variables are presented separately for ST-segment elevation myocardial infarction (STEMI) and for other ACS patients. In Finland, $36 \%$ of STEMI patients and 25\% of other ACS patients were admitted to hospitals having an emergency PCI service. The corresponding numbers for Norway were $77 \%$ and $66 \%$. However, the percentage of patients receiving PCI within one day was similar (STEMI: Norway 54\% vs. Finland $56 \%, \mathrm{p}<0.001$ ), as was the distribution of PCIs performed during weekends $(28 \%$ vs. $26 \%, p=0.02)$. The short term mortality was a little lower in Norway for STEMI patients (30-day mortality: $10 \%$ vs. $12 \%, \mathrm{p}<0.001 ; 365$-day mortality: $18 \%$ vs. $18 \%, \mathrm{p}=0.48)$, while markedly lower for other ACS (30-day mortality: $6 \%$ vs. $10 \%, \mathrm{p}<0.001 ; 365$-day mortality: $14 \%$ vs. $20 \%, p<0.001)$. After adjusting for individual and regional variables, the mortality was found to be $2-4 \%$ lower in Norway within most categories of the hospital system and organization of care variables in all analyses. As such, we were not able to explain the mortality differences by the hospital system and organization of care variables. Rather, the explanation seems to have other sources.
\end{abstract}

JEL classification: H11, I18

Key words: acute myocardial infarction, unstable angina pectoris, STEMI patients, PCI, care pathways, Finland, Norway

* Correspondence to: Terje P. Hagen, Department of Health Management and Health Economics, University of Oslo, P.O. Box 1089, 0318 Oslo, Norway. E-mail: t.p.hagen@medisin.uio.no.

Published: Online December 2018. In print January 2019. dx.doi.org/10.5617/njhe.4834 


\section{Introduction}

Mortality differences following hospital treatment in Finland and Norway are similar for stroke, hip fracture, and other major health problems (Häkkinen et al., 2015). Patients hospitalized with acute coronary syndrome (ACS) do, however, stand out as an important exception, with mortality in Finland being significantly higher than in Norway (Hagen et al., 2015). ACS refers to any group of symptoms indicating acute myocardial ischemia, and covers unstable angina pectoris, non-ST-segment elevation myocardial infarction (nonSTEMI), and ST-segment elevation myocardial infarction (STEMI). Of these, STEMI is the most severe, and is detected using electrocardiography graphs. Non-STEMI and unstable angina pectoris are closely related conditions, with non-STEMI being the more severe. NonSTEMI can be diagnosed by means of biomarkers (cardiac-specific troponins and creatine kinase). Such biomarkers cannot be detected in the bloodstream of unstable-angina patients (Thygesen et al., 2012).

Although some international comparisons of ACS/AMI mortality have been done previously, these either focus on demographic, clinical and treatment variables (Chung et al., 2014, McNamara et al., 2014), or have a North-American perspective (Ko et al., 2007). Studies focusing on comparisons of hospital system and organization variables and their impact on outcomes within Europe seem to be lacking.

Basic features of the health systems in Finland and Norway are relatively similar. There are, however, important differences in the organization of the treatment of ACS patients between the two countries, which might affect mortality. Finland and Norway both have tax-based health systems. In both countries, regional entities play a significant role in health care decision-making (Ringard et al., 2013, Vuorenkoski et al., 2008). The differences in the organization of treatments following ACS admissions are striking, however. As percutaneous coronary intervention (PCI) gradually became the preferred treatment for ACS patients during the 1990s, the Norwegian health regions decided to set up seven PCI facilities with acute services available 24 hours 7 days a week. Five of the seven PCI facilities were located at university hospitals and two were located at central hospitals. Prehospital services, including helicopter services, were expanded. In the Finnish health care system, decision making is decentralized to hospital districts and most of them have invested in their own PCI facilities, 20 PCI centers altogether in university hospitals and central hospitals. Due to high costs, only two of the PCI centers, both located at university hospitals, had formally established availability of acute services available 24/7 in 2009. By 2014, three others (two of them university hospitals) had formally established availability of acute services. The remaining hospitals had performed PCI under different arrangements, varying from acute services available $24 / 7$ on a voluntary basis to services available only during daytime on weekdays.

Guidelines developed by the European Society of Cardiology (ESC) for the most severe of the ACS patients, patients suffering STEMI, recommended less than 90 minutes from the onset of chest pain to the start of PCI treatment, which is the preferred treatment for most of these cases (Steg et al., 2012). The guidelines assume that the patient calls an emergency medical system (EMS) as soon as possible after the onset of chest pain. The EMS dispatches a fully equipped ambulance with personnel trained to perform and interpret electrocardiograms (ECG). Once the ECG reveals ST-segment elevation, the nearest PCI hospital is informed of the expected time of the patient's arrival. In cases where the diagnostic ECG has been done elsewhere (e.g., in a non-PCI hospital or at a physician's office), the EMS is called for transfer. According to the ESC, this scenario is best accomplished in a regional network with one high-volume PCI center, several surrounding 
non-PCI hospitals, and a single regional EMS. This sort of networks resembles the Norwegian model.

The main objectives of the study are twofold. First, to study how the differences in the organization of the treatment for ACS affect the treatment pathways for ACS patients, with a specific focus on patient and treatment volumes, travel time, transfers, and acute preparedness of the hospitals. Second, to analyze the association between these variables and the observed mortality differences.

\section{Data and methods}

\subsection{Data sources, inclusion criteria, and exclusion criteria}

Our database included patients registered in the Finnish and Norwegian national discharge registers hospitalized with ACS (International Classification of Diseases - ICD10 - I200, I21-I22) as the main diagnosis from 1 Jan. 2009 through 30 Nov. 2014. We developed a common protocol for variable definitions, inclusion criteria, and exclusion criteria used in both countries and linked data from the discharge registers to data from the causes of death registers (Häkkinen et al., 2013) (see also http://www.eurohope.info/doc/EHDP5_ AMI.pdf). The index admission is defined as the first hospitalization in a calendar year. To reduce heterogeneity in the patient population, we excluded patients if they had a known hospital admission with a main diagnosis of ACS within 365 days prior to the index admission. This means that we had access to data from 1 Jan 2008. For patients with multiple admissions more than a year apart, the first admission was used (5\% of the total sample). We further excluded patients who had no national ID number or known place of residence in the two countries. In the Finnish data, we further excluded patients treated only in health centers that lacked a specialty status (approximately 1000 patients per year) because these patients are older and have higher mortality than do acute hospital patients. We also excluded patients treated only in specialized health centers and private hospitals (approx. 100 patients during the data period).

Other data sources included Google Maps (travel time), Statistics Finland and Statistics Norway (demographic variables, the length of individual education, the level of individual income), the Finnish and Norwegian medical associations (number of cardiologist), and Eurostat and OECD (regional gross domestic products - GDP).

\subsection{Variables}

The outcome variables are 30-day and 365-day all-cause mortality following the date of the index admission.

Single stays were merged into episodes if subsequent discharge and admission dates were $\leq 1$ day. Hospitals were categorized according to local, regional, and university status, and the hospital at the highest level within the first seven days during the episode was defined as the episode hospital. Exact travel times and whether a helicopter was used (or not) were not available at the individual level. Instead, proxy travel times by car were calculated using travel distances between the patient's home municipality to the GPS coordinates of the first hospital as well as the episode hospital. In Norway, if travel time by car exceeds 30 minutes, helicopter transport will be used in case of emergencies. Hence, travel time to the first and the episode hospitals was categorized into $0<30 \mathrm{~min}$., $30<60$ min., and $\geq 60 \mathrm{~min}$. to account for this in the analyses. The categorization should reduce misclassification with respect to both proxy vs. real travel time and helicopter admissions: The likelihood of being transported by helicopter should increase across the categories for Norway, while remaining low in Finland, where a helicopter is rarely used. The volume of 
ACS patients in total per year and the volume of PCIs performed per year at the first and the episode hospitals were constructed by simple counts.

Analyses were done separately for STEMI patients (ICD-10: I210, I211, I212, I213, I220, I221, I228 and I229) and other ACS patients (ICD-10: I200, I219 and I214) defined by the discharge diagnosis of the first hospital treating the patient. STEMI coding was identified using common guidelines and clinical definitions in Norway and Finland and should as such be directly comparable. Hospitals in both countries follow the diagnostic guidelines of the ESC (Thygesen et al., 2012, Hamm et al., 2011). Diagnosis were registered at discharge. The quality of the hospital discharge registers are regarded as good (Sund 2012, Bakken et al., 2014). Still, it is hard completely to dismiss the possibility that differences exist because of diagnostic coding practices. We regarded the classification of the "other ACS patients", which included non-STEMI, undefined acute myocardial infarction (AMI) and unstable-angina patients, as less precise and analyzed these subgroups separately.

Four sets of variables in different combinations are used to describe the hospital system and organization of care:

- Indicators on PCI treatment (yes/no).

- Whether the admission was during the weekend or not.

- Hospital variables included indicator variables on acute preparedness for PCI (PCI available permanently 24/7, thus not including Finnish voluntary centers), PCI volume per year, and ACS volume per year for the episode hospital.

- Transfers during a hospital episode.

There is some uncertainty related to the coding of the hospitals in Finland having voluntary solutions. We handle this uncertainty by using alternative coding in several sensitivity analyses.

As control variables we included age, gender, and gross individual income in the year prior to the index admission, highest attained education at admission, and the total length of stay for all non-ACS hospital admissions in the previous year. Income was adjusted to the 2014 level by using national consumer price indices, and Norwegian income was adjusted to Finnish income using purchasing power parities. Regional variables included population density per km2, GDP per capita, the share of the population above 80 years, and the number of cardiologists per 100,000 inhabitants - all calculated per year. Previous analyses (Häkkinen et al, 2015) have indicated that living in rural regions with fewer resources is associated with mortality for both AMI and other diagnoses, but to different degrees in Norway and Finland. The regions correspond to hospital districts (Finland) and health enterprises (Norway), classified according to the home municipality of the patient. In both countries, the regions approximated the counties. Regional GDP per capita for Finland was collected from OECD regional databases (OECD Regional Statistics http://www.oecd-ilibrary.org/urban-rural-and-regional-development/data/oecd-regionalstatistics_region-data-en) and for Norway from OECD and Statistics Norway. The GDP per capita figures were changed to US\$ as follows: The regional figures from both countries from the year 2014 were changed to US\$ using GDP purchasing power parities (PPP) from the year 2014. Earlier-year regional figures were first changed to the 2014 price level using national GDP price indices and then changed to US\$ using GDP PPP from the year 2014. 


\subsection{Statistical analyses}

Descriptive statistics are shown as means for continuous variables and as percentages for categorical variables. For the continuous regional and hospital variables, these will be weighted means because they are calculated using the individual data. Hence, tables show the population density, GDP, the proportion above 80 years, cardiologists per 100,000, and the number of ACS/PCI performed per year for the region/hospital treating the average patient and are as such very different from, for example, the population density of the country or the average volume per hospital. P-values indicating differences between countries were calculated using t-tests for the continuous variables and chi-square tests for the categorical variables.

Regression models for marginal effects of hospital system and organization of care variables

To analyze the association between hospital system and organization of care variables (hereafter called "system and care variables") and any observed mortality differences, logistic regression models were used. We focus on the following variables: the distance to the episode hospital (categorized as mentioned above) and indicators of transfer within the episode, the index admission day (weekends or not), treatment at a PCI 24/7 hospital during the episode, and PCI treatment within one day from the index admission day. Treatment at a PCI 24/7 hospital both captures the largest hospitals in both countries and is directly linked to the different organization of care in the countries. The following specification was used:

$$
\begin{aligned}
\operatorname{logit}\left(p_{\text {death }}\right)= & \beta_{0}+\beta_{1} \text { Fin }+\beta_{2} \text { Dist }_{30-60}+\beta_{3} \text { Dist }_{>60}+\beta_{4} \text { Transfer }+\beta_{5} \text { PCI }_{1 \text { day }} \\
& +\beta_{6} \text { PCI } 24 / 7+\beta_{7} \text { Weekend }+\beta_{8} \text { PCI } 24 / 7 \cdot \text { Weekend }+\beta_{12} \text { Fin }_{\text {Dist }} \text { W0-60 } \\
& +\beta_{13} \text { Fin } \cdot \text { Dist } \\
& +60+\beta_{14} \text { Fin } \cdot \text { Transfer }+\beta_{15} \text { Fin } \cdot \text { PCI }_{1 \text { day }}+\beta_{16} \text { Fin } \cdot \text { PCI24/7 } \\
& +\beta_{17} \text { Fin } \cdot \text { Weekend }+\beta_{18} \text { Fin } \cdot \text { PCI } 24 / 7 \cdot \text { Weekend }
\end{aligned}
$$

To capture effects due to less preparedness during weekends in Finland for patients admitted to non-24/7 hospitals, the indicators for PCI 24/7 episode admission and index admission day during weekends are included in an interaction term. Interactions by country (Fin) were included to allow for different effects of the variables in Norway and Finland. Because the selection of patients transferred to larger hospitals may differ between the countries, analyses are done separately for all patients and for patients without transfers only (excluding $\beta_{4}$ and $\beta_{14}$ ). The latter should to a greater degree include patients who are treated locally (i.e., within the hospital district/enterprise) and should therefore be less vulnerable to different selection effects related to hospital volume, although in Norway, patients may still be admitted directly to a PCI hospital not within the catchment area of their local health enterprise.

Whether any mortality difference is related to the system and care variables is captured by the interaction coefficients $\beta_{12}, \beta_{13}, \ldots, \beta_{18}$ in the model. If $\beta_{14}=0$, it means that the effect of transferring patients is equal in both countries; hence, the transfer variable does not seem to explain any mortality difference. A test for the effect of acute preparedness is to study whether the effect of the four combinations of PCI24/7-weekend differ across countries (one or more of $\beta_{16}, \beta_{17}, \beta_{18} \neq 0$ ).

Results are presented as marginal mortality differences in percentage points between Norway and Finland for each category of the system and care variables (e.g., no transfer/transfer). This is interpreted as the adjusted effect of being Norwegian rather than Finnish if all patients belonged to the respective category of the system and care variables (e.g., no transfer), and if all other variables in the model were as observed. Again, a 
relationship to a system and care variable would be indicated by non-constant mortality differences across categories (e.g., a smaller mortality difference for non-transferred than for transferred patients). A negative difference means that the adjusted marginal mortality is lower in Norway than in Finland, while a positive difference indicates the opposite. We have included $\mathrm{p}$-values for the marginal differences, and have marked where the interaction coefficients $\beta_{12}, \beta_{13}, \ldots, \beta_{18}$ are significant at the $5 \%$ level.

Eight models are presented: 30-day (short-term, data period 1 Jan. 2009-30 Nov. 2014) and 365-day (long-term, data period 1 Jan. 2009-31 Dec. 2013) mortality, separately for all STEMI patients and STEMI patients without transfers only, and for other ACS patients and other ACS patients without transfers only.

Not included in the equation above to save space, models were additionally adjusted for the regional variables population density, GDP per capita, percentage of population above 80 years and cardiologists per 100,000 (included as continuous variables). We also included the individual variables gender, age, average income in the year prior to the index admission, highest attained education at admission, length of hospital stays in the previous year and type of ACS. Calendar year dummy variables were included as well. Age was categorized into ten categories starting at $<49$ years, then in 5-year intervals up to 89 years, and finally $>90$ years. Income was included as continuous, while education was categorized into primary, secondary, and tertiary levels. The length of stay in the previous year was categorized into 0 days, 1-10 days, and > 10 days due to skewed distributions. Interactions by country for these variables were included if significant at the $5 \%$ level from partial Ftests.

Robust standard errors were used, accounting for clustering at the episode-hospital level. No significant clustering effect was found at the regional level, using multilevel random effects logistic regression. Data management was done by SAS version 9.4 and data analysis by Stata version 14.

\section{Results}

Table 1, left, shows descriptive statistics for the STEMI patients. The mortality differences are small, but statistically significant for 30-day mortality because of the large samples. The variables describing the system and care clearly show a number of important differences in the organization of care. The average Norwegian patient is living further from the hospital, has a greater likelihood of being transferred during the episode, and is treated at a hospital with much higher volumes of ACS patients and PCIs performed per year and with better acute preparedness measured by PCI available 24/7 than does the average Finnish patient. The standard deviations of the continuous hospital variables are also considerably larger in Norway. Still, this does not influence the probability of receiving PCI within the first day and influences only to a small degree the probability of receiving PCI during the weekend.

Table 1, right, shows the corresponding results for the other ACS patients. The mortality in Norway is markedly lower for this group. The proportion of unstable angina cases is similar in both countries, but Norway has a higher proportion recorded as undefined AMI and Finland has a corresponding higher proportion of non-STEMI patients. The variables describing the system and care show large differences also for the group of other ACS patients: In Norway, there is a much greater likelihood than in Finland of being transferred during the episode and treated at a hospital with higher volumes of ACS patients and PCIs performed per year and with better preparedness. This greater likelihood applies even though a minority of patients receives PCI. Hence, the results indicate that in Norway, the other ACS patients are still treated at the large hospitals, either by being sent there directly or by being transferred from a local hospital. 
Table 1: Descriptive statistics for the STEMI and other ACS patients (non-STEMI, undefined AMI, and unstable angina pectoris)

\begin{tabular}{|c|c|c|c|c|c|c|}
\hline & STEMI & & & Other ACS & & \\
\hline Outcomes: & $\begin{array}{l}\text { Norway } \\
(\mathrm{n}=18,747)\end{array}$ & $\begin{array}{l}\text { Finland } \\
(\mathrm{n}=17,167)\end{array}$ & $\mathrm{p}$-value & $\begin{array}{l}\text { Norway } \\
(\mathrm{n}=49,711)\end{array}$ & $\begin{array}{l}\text { Finland } \\
(\mathrm{n}=38,798)\end{array}$ & p-value \\
\hline$\%$ 30-day mortality & 10 & 12 & $<0.001$ & 6 & 10 & $<0.001$ \\
\hline$\%$ 365-day mortality (total n)* & $18(16,491)$ & $18(14,575)$ & 0.41 & $14(45,067)$ & $20(34,244)$ & $<0.001$ \\
\hline \multicolumn{7}{|l|}{ Individual variables: } \\
\hline$\%$ males & 68 & 67 & 0.04 & 62 & 61 & $<0.01$ \\
\hline Age (SD) & $69.2(14.1)$ & $68.1(13.2)$ & $<0.001$ & $70.9(13.7)$ & $72.3(12.2)$ & $<0.001$ \\
\hline $\begin{array}{l}\text { LOS (days) in previous year } \\
\text { (SD) }\end{array}$ & $3.2(9.7)$ & $2.9(6.5)$ & $<0.01$ & $4.0(10.4)$ & $4.2(8.9)$ & $<0.001$ \\
\hline $\begin{array}{l}\text { Income (EUR) } \\
\text { (SD) }\end{array}$ & $\begin{array}{l}32,500 \\
(25,200)\end{array}$ & $\begin{array}{l}23,200 \\
(20,300)\end{array}$ & $<0.001$ & $\begin{array}{l}31,400 \\
(24,000)\end{array}$ & $\begin{array}{l}20,800 \\
(17,400)\end{array}$ & $<0.001$ \\
\hline$\%$ primary education & 40 & 53 & $<0.001$ & 40 & 59 & $<0.001$ \\
\hline$\%$ secondary education & 46 & 31 & & 45 & 26 & \\
\hline$\%$ tertiary education & 14 & 16 & & 15 & 15 & \\
\hline$\%$ non-STEMI & & & & 39 & 57 & $<0.001$ \\
\hline$\%$ undefined & & & & 33 & 18 & \\
\hline$\%$ unstable angina & & & & 28 & 25 & \\
\hline Regional variables: & 19 regions & 19 regions & & 19 regions & 19 regions & \\
\hline Pop. density per km2 (SD) & $120(300)$ & $55(64)$ & $<0.001$ & $106(280)$ & $46(57)$ & $<0.001$ \\
\hline GDP per capita (EUR) (SD) & $\begin{array}{l}61,000 \\
(19,000)\end{array}$ & $\begin{array}{l}40,000 \\
(9,000)\end{array}$ & $<0.001$ & $\begin{array}{l}61,000 \\
(17,000)\end{array}$ & $\begin{array}{l}39,000 \\
(8,000)\end{array}$ & $<0.001$ \\
\hline$\%$ above age 80 years & $4.5(0.7)$ & $5.1(1.0)$ & $<0.001$ & $4.5(0.7)$ & $5.2(1.0)$ & $<0.001$ \\
\hline $\begin{array}{l}\text { Cardiologists per } 100,000 \\
\text { (SD) }\end{array}$ & $5.3(2.9)$ & $3.9(1.7)$ & $<0.001$ & $5.1(2.8)$ & $4.0(1.8)$ & $<0.001$ \\
\hline System and care variables: & $\begin{array}{l}52 \text { hospitals } \\
320 \text { ACS } \\
\text { patients/year }\end{array}$ & $\begin{array}{l}32 \text { hospitals } \\
317 \text { ACS } \\
\text { patients/year }\end{array}$ & & $\begin{array}{l}52 \text { hospitals } \\
320 \text { ACS } \\
\text { patients/year }\end{array}$ & $\begin{array}{l}32 \text { hospitals } \\
317 \text { ACS } \\
\text { patients/year }\end{array}$ & \\
\hline $\begin{array}{l}\text { Distance to first hospital, } \\
\text { minutes (SD) }\end{array}$ & $69(99)$ & $49(71)$ & $<0.001$ & $57(83)$ & $38(41)$ & $<0.001$ \\
\hline$\%$ transferred during episode & 16 & 9 & $<0.001$ & 37 & 8 & $<0.001$ \\
\hline $\begin{array}{l}\text { Distance to episode hospital, } \\
\text { min. (SD) }\end{array}$ & $93(125)$ & $51(71)$ & $<0.001$ & $98(133)$ & $43(20)$ & $<0.001$ \\
\hline $\begin{array}{l}\% \text { treated at PCI } 24 / 7 \text { hospital } \\
\text { during episode }\end{array}$ & 77 & 36 & $<0.001$ & 66 & 25 & $<0.001$ \\
\hline$\%$ PCI within 1 day & 55 & 56 & $<0.001$ & 18 & 24 & $<0.001$ \\
\hline $\begin{array}{l}\% \text { of PCI performed during } \\
\text { weekend }\end{array}$ & 28 & 26 & 0.02 & 18 & 19 & 0.03 \\
\hline $\begin{array}{l}\text { ACS hospital volume per year } \\
\text { (SD) }\end{array}$ & $\begin{array}{l}1,360 \\
(1,009)\end{array}$ & $715(470)$ & $<0.001$ & $\begin{array}{l}1,190 \\
(1,014)\end{array}$ & $580(415)$ & $<0.001$ \\
\hline $\begin{array}{l}\text { PCI hospital volume per year } \\
\text { (SD) }\end{array}$ & $\begin{array}{l}1,613 \\
(1,429)\end{array}$ & $802(609)$ & $<0.001$ & $\begin{array}{l}1,350 \\
(1,405)\end{array}$ & $630(563)$ & $<0.001$ \\
\hline $\begin{array}{l}\% \text { treated in university } \\
\text { hospitals }\end{array}$ & 71 & 51 & $<0.001$ & 61 & 42 & $<0.001$ \\
\hline
\end{tabular}

Note: Data from 1 Jan. 2009-30 Nov. 2014, including p-values for differences. *For 365-day mortality, 1 Jan. 2009-31 Dec. 2013 is used. LOS = length of stay, SD = standard deviation. 
Table 2 shows the between-country difference in mortality for the variables describing the system and care variables for the STEMI patients. For example for 30-day mortality, patients living less than 30 minutes from hospital by car have 4.0 percentage points lower mortality in Norway than Finland after adjustment for system and care variables, background variables and interactions. Patients admitted during weekdays to nonPCI24/7 hospitals have 3.5\% lower mortality in Norway than Finland. For 30-day mortality, only patients admitted to PCI 24/7 hospitals during weekends do not show a significantly lower mortality in Norway than in Finland. This applies in both analyses of all patients and analyses of patients without transfer only, but seems to be a result of the smaller sample sizes for these groups because the effects are similar in magnitude to the effects of the other categories. For 365-day mortality, most differences between the countries are smaller, and not always significant. There are no signs of a weekend effect for the STEMI patients, because no interactions are significant (indicated by lack of * - see table text for explanation), and differences during the weekend are only marginally larger than differences during weekdays. For patients not treated by PCI within 1 day the 30-day mortality is 3.2\% lower in Norway than in Finland and for patients receiving PCI within 1 day 30-day mortality is $3.6 \%$ lower in Norway than in Finland. In this case the interaction effect is significant (indicated by the *), meaning that the mortality difference between the countries for those receiving PCI within 1 day is higher than for those that do not receive treatment within 1 day. There are also no signs of increasing differences across the categories of travel time to the episode hospital.

Table 3 shows the corresponding results for the other ACS patients. For 30-day mortality, all categories show lower mortality in Norway than in Finland in both analyses of all patients and analyses of patients without transfers only. For 365-day mortality, there is no difference in mortality when comparing patients receiving PCI within one day, and the difference for patients admitted to PCI 24/7 hospitals during weekends is only borderline significant. However, in the analysis of patients without transfers only, patients admitted to PCI 24/7 hospitals during weekdays also have similar mortality. There are indications of an increased difference in mortality for patients admitted during the weekend in both hospital categories, but these interaction coefficients are not significant. Having a transfer during the episode significantly increases the mortality difference compared with patients without a transfer. Also for other ACS patients, there are no indications of an increased mortality difference for longer distances to the episode hospital.

So far, Finnish hospitals were categorized according to permanent 24/7 availability because this is most comparable to the Norwegian organization. As sensitivity analyses, we experimented with different coding of the voluntary PCI hospitals in Finland. The results were, however, similar across the analyses; hence, only the basic versions of the models are reported.

\section{Discussion}

In Finland, patients are treated locally in smaller units, whereas in Norway, patients are transported for long distances and treated at centralized units. This difference applies both to STEMI patients and to group of other ACS patients, where only a minority will receive PCI. The organization of care that we find in Norway closely resembles the organization of care that is recommended by the European Society of Cardiology (ECS).

However, contrary to our expectations, the differences in organization of care do not influence the likelihood of receiving PCI, and the effect of the system and care variables appear to be similar because few significant interactions by country are found. Hence in that sense, both countries' organizations produce similar results. An interesting finding for 
Table 2: Marginal mortality differences in percentage points, Norway (NOR) vs. Finland (FIN) and p-values (p), for different categories of system and care variables for the STEMI patients

\begin{tabular}{|c|c|c|c|c|}
\hline \multicolumn{5}{|l|}{ All STEMI patients } \\
\hline \multirow{3}{*}{$\begin{array}{l}\text { System and care variables: } \\
\text { Total travel time } 0<30 \text { minutes (Ref) }\end{array}$} & \multirow{2}{*}{\multicolumn{2}{|c|}{$\begin{array}{l}\text { 30-day mortality } \\
\text { NOR vs. FIN (p) }\end{array}$}} & \multirow{2}{*}{\multicolumn{2}{|c|}{$\begin{array}{l}\text { 365-day mortality } \\
\text { NOR vs. FIN (p) }\end{array}$}} \\
\hline & & & & \\
\hline & $-4.0 \%$ & $<0.001$ & $-2.1 \%$ & 0.04 \\
\hline Total travel time $30<60$ minutes & $-3.9 \%$ & $<0.001$ & $-3.1 \%$ & 0.02 \\
\hline Total travel time $\geq 60$ minutes & $-3.5 \%$ & $<0.001$ & $-2.5 \%$ & 0.03 \\
\hline Admit during weekdays, episode hospital non-PCI 24/7 (Ref) & $-3.5 \%$ & $<0.001$ & $-2.4 \%$ & 0.02 \\
\hline Admit during weekend, episode hospital non-PCI 24/7 & $-3.5 \%$ & $<0.001$ & $-1.6 \%$ & 0.23 \\
\hline Admit during weekdays, episode hospital PCI 24/7 & $-4.2 \%$ & $<0.01$ & $-2.7 \%$ & 0.11 \\
\hline Admit during weekend, episode hospital PCI 24/7 & $-4.4 \%$ & 0.08 & $-3.3 \%$ & 0.12 \\
\hline No PCI within 1 day (Ref) & $-3.6 \%$ & 0.01 & $-1.8 \%$ & 0.27 \\
\hline PCI within 1 day & $-3.2 \% *$ & $<0.001$ & $-3.2 \% *$ & $<0.001$ \\
\hline No transfer within episode (Ref) & $-3.8 \%$ & $<0.001$ & $-2.3 \%$ & 0.05 \\
\hline Transfer to higher-level hospital within episode & $-4.1 \%$ & $<0.001$ & $-3.9 \%$ & 0.01 \\
\hline $\mathrm{N}$ & 35,874 & & 31,034 & \\
\hline Pseudo $\mathrm{R}^{2}$ & 0.18 & & 0.24 & \\
\hline Area under the curve & 0.81 & & 0.83 & \\
\hline \multicolumn{5}{|c|}{ STEMI patients without transfers only } \\
\hline Total travel time $0<30$ minutes (Ref) & $-4.1 \%$ & $<0.001$ & $-1.8 \%$ & 0.10 \\
\hline Total travel time $30<60$ minutes & $-3.8 \%$ & $<0.01$ & $-2.7 \%$ & 0.07 \\
\hline Total travel time $\geq 60$ minutes & $-3.2 \%$ & $<0.01$ & $-2.0 \%$ & 0.11 \\
\hline Admit during weekdays, episode hospital non-PCI 24/7 (Ref) & $-3.8 \%$ & $<0.001$ & $-2.6 \%$ & 0.01 \\
\hline Admit during weekend, episode hospital non-PCI 24/7 & $-3.8 \%$ & $<0.001$ & $-2.0 \%$ & 0.16 \\
\hline Admit during weekdays, episode hospital PCI 24/7 & $-3.6 \%$ & 0.04 & $-1.0 \%$ & 0.56 \\
\hline Admit during weekend, episode hospital PCI 24/7 & $-4.2 \%$ & 0.15 & $-2.2 \%$ & 0.35 \\
\hline No PCI within 1 day (Ref) & $-3.2 \%$ & 0.04 & $-0.8 \%$ & 0.63 \\
\hline PCI within 1 day & $-3.6 \% *$ & $<0.001$ & $-3.3 \% *$ & $<0.001$ \\
\hline $\mathrm{N}$ & 31,372 & & 26,927 & \\
\hline Pseudo $\mathrm{R}^{2}$ & 0.18 & & 0.24 & \\
\hline Area under the curve & 0.80 & & 0.83 & \\
\hline
\end{tabular}

* indicates a significant interaction regression coefficient for the given category compared to the reference category (Ref) at the 5\% level. All estimates are adjusted for individual variables (gender, age, length of stay the previous year, income, education, calendar year) and regional variables (population density, GDP per capita, \% population $>80$ years, cardiologists per 100,000), including significant interactions by country.

Norway is that a majority of STEMI patients seem to be sent directly to the large-volume hospitals (hence there is a low proportion of transfers), while the group of other ACS patients are sent to the large hospitals via transfer from a local hospital. In summary, this leads to high percentages being treated at 24/7 PCI hospitals for both STEMI and other ACS patients in Norway, which is very different from Finland. 
Table 3: Marginal mortality differences in percentage points, Norway (NOR) vs. Finland (FIN) and p-values (p), for different categories of system and care variables for the other $\mathrm{ACS}$ patients

All other ACS patients

\begin{tabular}{|c|c|c|c|c|}
\hline \multirow{3}{*}{$\begin{array}{l}\text { System and care variables: } \\
\text { Total travel time } 0-30 \text { minutes (Ref) }\end{array}$} & \multirow{2}{*}{\multicolumn{2}{|c|}{$\begin{array}{l}\text { 30-day mortality } \\
\text { NOR vs. FIN (p) }\end{array}$}} & \multirow{2}{*}{\multicolumn{2}{|c|}{$\begin{array}{l}\text { 365-day mortality } \\
\text { NOR vs. FIN (p) }\end{array}$}} \\
\hline & & & & \\
\hline & $-3.0 \%$ & $<0.001$ & $-3.1 \%$ & $<0.001$ \\
\hline Total travel time $30-60$ minutes & $-3.3 \%$ & $<0.001$ & $-3.6 \%$ & $<0.001$ \\
\hline Total travel time $>60$ minutes & $-3.7 \%$ & $<0.001$ & $-3.4 \%$ & $<0.001$ \\
\hline Admit during weekdays, episode hospital non-PCI 24/7 (Ref) & $-3.5 \%$ & $<0.001$ & $-3.6 \%$ & $<0.001$ \\
\hline Admit during weekend, episode hospital non-PCI 24/7 & $-3.7 \%$ & $<0.001$ & $-4.1 \%$ & $<0.001$ \\
\hline Admit during weekdays, episode hospital PCI $24 / 7$ & $-2.5 \%$ & $<0.001$ & $-2.4 \%$ & 0.01 \\
\hline Admit during weekend, episode hospital PCI 24/7 & $-3.5 \%$ & $<0.001$ & $-2.5 \%$ & 0.13 \\
\hline No PCI within 1 day (Ref) & $-3.5 \%$ & $<0.001$ & $-3.8 \%$ & $<0.001$ \\
\hline PCI within 1 day & $-1.5 \%$ & $<0.01$ & $-0.2 \% *$ & 0.77 \\
\hline No transfer within episode (Ref) & $-3.2 \%$ & $<0.001$ & $-2.9 \%$ & $<0.001$ \\
\hline Transfer to higher-level hospital within episode & $-3.9 \% *$ & $<0.001$ & $-6.1 \% *$ & $<0.001$ \\
\hline $\mathrm{N}$ & 88,445 & & 75,210 & \\
\hline Pseudo $\mathrm{R}^{2}$ & 0.21 & & 0.25 & \\
\hline Area under the curve & 0.84 & & 0.84 & \\
\hline
\end{tabular}

Other ACS patients without transfers only

\begin{tabular}{|c|c|c|c|c|}
\hline Total travel time $0-30$ minutes (Ref) & $-3.4 \%$ & $<0.001$ & $-3.1 \%$ & $<0.01$ \\
\hline Total travel time $30-60$ minutes & $-3.7 \%$ & $<0.001$ & $-3.6 \%$ & $<0.01$ \\
\hline Total travel time $>60$ minutes & $-4.2 \%$ & $<0.001$ & $-3.4 \%$ & $<0.01$ \\
\hline Admit during weekdays, episode hospital non-PCI 24/7 (Ref) & $-3.9 \%$ & $<0.001$ & $-3.7 \%$ & $<0.001$ \\
\hline Admit during weekend, episode hospital non-PCI 24/7 & $-4.1 \%$ & $<0.001$ & $-4.2 \%$ & $<0.001$ \\
\hline Admit during weekdays, episode hospital PCI 24/7 & $-2.5 \% *$ & $<0.001$ & $-1.3 \% *$ & 0.23 \\
\hline Admit during weekend, episode hospital PCI 24/7 & $-3.8 \%$ & $<0.01$ & $-2.6 \%$ & 0.20 \\
\hline No PCI within 1 day (Ref) & $-3.8 \%$ & $<0.001$ & $-3.6 \%$ & $<0.001$ \\
\hline PCI within 1 day & $-2.4 \%$ & $<0.001$ & $-1.3 \%$ & 0.14 \\
\hline $\mathrm{N}$ & 66,944 & & 57,329 & \\
\hline Pseudo $\mathrm{R}^{2}$ & 0.19 & & 0.22 & \\
\hline Area under the curve & 0.81 & & 0.82 & \\
\hline
\end{tabular}

* indicates a significant interaction regression coefficient for the given category compared to the reference category (Ref) at the 5\% level. All estimates are adjusted for individual variables (gender, age, length of stay the previous year, ICD-10 category, income, education, calendar year) and regional variables (population density, GDP per capita, \% population > 80 years, cardiologists per 100,000), including significant interactions by country.

Additional analyses of the marginal country differences in the models indicated that the crude mortality differences for the group of other ACS patients could not be explained by the variables used in our analyses. Even after including all variables in the models, the marginal country differences were still highly significant, with $3.4 \%-4.0 \%$ lower mortality in Norway across the models. For the STEMI patients, the crude mortality difference was 
small, as seen in Table 1. However, the difference increased after adjustment for the individual variables because Finland has more patients in the younger age groups and a shorter length of stay in the year prior to the index admission than does Norway. This difference did not disappear after adjustment for regional and system and care variables.

Even though there are omitted variables (e.g. on lifestyle and comorbidities) that would explain more of the observed mortality differences, we still argue that the current analysis is informative for evaluating the impact of the system and care variables. For instance, the mortality difference between Norway and Finland could be due to the better health of Norwegian patients. However, unless the health factors are associated with weekday of admission, travel time, and acute preparedness (confounding), and the strengths of the associations differ between the countries (interaction), the mortality difference across categories of a system and care variable would still be constant. The strength of the confounding and interaction effects would also need to be substantial, because the organizational differences were so large (Table 1). Thus, any non-constant differences across categories of a system and care variable should indicate an association to organization and/or treatment differences, not health differences. Still, one would have to do further analyses to identify whether different system selection mechanisms or treatment approaches not covered here were the explanations (exact time to PCI, approaches to rehabilitation, medication, etc.).

Another challenge is selection effects associated with the system and care variables that differed between the countries. Patients who are too fragile to undergo PCI or other significant treatment may be treated in smaller hospitals throughout the episode. This approach would lead to an apparent effect of high volume on mortality, but is in reality a selection effect due to severe patients being over-represented in smaller hospitals. Patients in Norway are treated in larger, more centralized hospitals than are patients in Finland, which could indicate differences in selection effects, leading to bias in the analysis. However, this is a problem only when the difference in selection effects between countries depends on weekday of admission, and we also analyzed patients without transfers separately to further reduce the risk of bias. In the model, this is captured by the interaction term between PCI24/7 and the weekend indicator. Hence, if the mortality difference for PCI24/7 patients changes significantly between patients admitted on weekends and weekdays, it should indicate an effect related to the system/organization of care. We do not find that.

Still, there were some interesting results from the analyses when both the STEMI and other ACS analyses are viewed in combination: Mortality differences were generally smaller for 365-day mortality than for 30-day mortality. The system and care variables should have a stronger effect in the short run than in the long run. The analyses of patients without transfers showed smaller differences between patients admitted to PCI 24/7 hospitals than the analyses of all patients. This difference is expected, because the former analyses should include more patients treated locally than the latter, and these hospitals should be the most comparable in terms of facilities. This is also reflected by the fact that the mortality difference was only borderline or not significant for STEMI patients admitted to 24/7 PCI hospitals (Table 2, patients without transfers). The differences were greater in the group receiving PCI within one day than in the group not receiving PCI within one day for the 365-day analysis of all STEMI patients and for both 30-day and 365-day mortality for STEMI patients without transfer. Although not shown in Table 2, the country-PCI interaction coefficient was significant and negative also for 30-day mortality in all STEMI patients, corresponding to a more protective effect of PCI in Norway than in Finland (estimated to $0.5 \%$ lower mortality from the model). These findings could indicate better selection of patients to PCI or quicker access to the procedure in Norway than in Finland. 
One day is a crude indicator in this setting. For the other ACS patients, the difference decreased for patients receiving PCI within one day compared with those not receiving PCI within one day (Table 3). Because these patients less frequently need the procedure and it is less important in their treatment, this result does not necessarily contradict the previous one. The other ACS patients also showed a larger mortality difference if they were transferred to a higher-level hospital during the episode than did patients without a transfer. This difference could indicate that only seriously ill patients are transferred in Finland, whereas a mixed group of patients are transferred in Norway. This is reflected by the fact that transfer is much more common for the group of other ACS patients in Norway than in Finland.

Contrary to our expectations, there were no indications of increased mortality differences with longer travel time. It could be that information on the mode of transportation and on the travel time at the individual level is necessary to identify an effect. Although the mortality differences where larger during weekends than during weekdays in all analyses, the increase was not significant in any of the models.

A further research topic of relevance to policy makers would be to investigate the cost effectiveness of the alternative organizational forms. If costs do not differ, the organizational form does not matter, but if the total costs of either system, including referrals, exceed the total costs of the other system, policy makers should carefully evaluate the options.

In conclusion, Norway and Finland differ greatly in their organization of care for ACS patients, and this difference has not been highlighted in previous research. Still, because the organizational aspects considered here do not explain much of the mortality difference, it is hard to say that one organization is better than the other. Rather, the explanation for the mortality difference seems to be found in other health and treatment factors.

\section{Acknowledgements}

The data gathering and analyses are funded by the Health Programme of the European Union (grant number 664691 / BRIDGE Health). Unto Häkkinen acknowledges financial support from the Yrjö Jahnsson Foundation and Finnish Society for Health Economics.

\section{References}

Bakken, I. J., P. Suren, S. E. Haberg, et al. (2014). [The Norwegian patient register--an important source for research]. Tidsskrift for den norske legeforening, 134(1), 12-3.

Chung, S. C., R. Gedeborg, O. Nicholas, et al. (2014). Acute myocardial infarction: a comparison of short-term survival in national outcome registries in Sweden and the UK. Lancet, 383(9925), 1305-12.

Hagen, T. P., U. Häkkinen, E. Belicza, et al. (2015). Acute Myocardial Infarction, Use of Percutaneous Coronary Intervention, and Mortality: A Comparative Effectiveness Analysis Covering Seven European Countries. Health Economics, 24 Suppl 2, 88-101.

Häkkinen, U., T. Iversen, M. Peltola, et al. (2013). Health care performance comparison using a disease-based approach: the EuroHOPE project. Health Policy, 112(1-2), 100-9.

Häkkinen, U., G. Rosenqvist, T. Iversen, et al. (2015). Outcome, Use of Resources and Their Relationship in the Treatment of AMI, Stroke and Hip Fracture at European Hospitals. Health Economics, 24 Suppl 2, 116-39. 
Hamm, C. W., J. P. Bassand, S. Agewall, et al. (2011). ESC Guidelines for the management of acute coronary syndromes in patients presenting without persistent ST-segment elevation: The Task Force for the management of acute coronary syndromes (ACS) in patients presenting without persistent ST-segment elevation of the European Society of Cardiology (ESC). European Heart Journal, 32(23), 2999-3054.

Ko, D. T., H. M. Krumholz, Y. Wang, et al. (2007). Regional differences in process of care and outcomes for older acute myocardial infarction patients in the United States and Ontario, Canada. Circulation 115(2), 196-203.

McNamara, R. L., S. C. Chung, et al. (2014). International comparisons of the management of patients with non-ST segment elevation acute myocardial infarction in the United Kingdom, Sweden, and the United States: The MINAP/NICOR, SWEDEHEART/RIKS-HIA, and ACTION Registry-GWTG/NCDR registries. International Journal of Cardiology, 175(2), 240247.

Ringard, Å., A. Sagan, I. Sperre Saunes et al. (2013). Norway: Health system review. Health Systems in Transition, 15(8), 1-162.

Steg, P. G., S. K. James, D. Atar, et al. (2012). ESC Guidelines for the management of acute myocardial infarction in patients presenting with ST-segment elevation: The Task Force on the management of ST-segment elevation acute myocardial infarction of the European Society of Cardiology (ESC). European Heart Journal, 33(20), 2569-619.

Sund, R. (2012). Quality of the Finnish Hospital Discharge Register: a systematic review. Scandinavian Journal of Public Health, 40(6), 505-15.

Thygesen, K., J. S. Alpert, A. S. Jaffe, et al. (2012). Third universal definition of myocardial infarction. European Heart Journal, 33(20), 2551-67.

Vuorenkoski L, Mladovsky P and Mossialos E. 2008. Finland: Health system review. Health Systems in Transition, 10(4), 1-168.

(C) 2018 by the author(s). This article is an open access article distributed under the terms and conditions of the Creative Commons Attribution license (http://creativecommons.org/licenses/by/4.0/). 